\title{
Trifluridine and Tipiracil Hydrochloride
}

\author{
National Cancer Institute
}

\section{Source}

National Cancer Institute. Trifluridine and Tipiracil Hydrochloride. NCI Thesaurus. Code C102554.

An orally bioavailable combination agent composed of the cytotoxic pyrimidine analog trifluridine (5-trifluoro-2'-deoxythymidine or TFT) and a thymidine phosphorylase inhibitor (TPI) tipiracil hydrochloride, in a molar ratio of 1.0:0.5 (TFT:TPI), with potential antineoplastic activity. After oral administration of TAS-102, TFT is phosphorylated to the active monophosphate form TF-TMP, which binds covalently to the active site of thymidylate synthase, thereby reducing the nucleotide pool levels required for DNA replication. Furthermore, the triphosphate form TF-TTP can be incorporated into DNA, which induces DNA fragmentation and leads to the inhibition of tumor growth. TPI exhibits a dual effect: 1) an anti-ang iogenic effect mediated through the inhibition of thymidine phosphorylase, which plays important role in nucleotide metabolism and a variety of development processes, including angiogenesis, 2 ) increased bioavailability of the normally short-lived antimetabolite TFT by preventing its degradation into the inactive form trifluorothymine (TF-Thy). The synergistic effect of the components in TAS-10 may demonstrate antitumor activity in 5-FU-resistant cancer cells. 